\title{
Image Processing of the Lumber Surface Defect Based on Morphology
}

\author{
YANG Jian-Hua ${ }^{1,2,3, a}$, FU Wan-Si²,b, XIAO Jiang ${ }^{3, c}$ and YU Miao ${ }^{2, d}$ \\ ${ }^{1}$ Forestry New Technology Research Institute, Chinese Academy of Forestry, Beijing, 100091, China \\ ${ }^{2}$ Beijing Forestry Machinery Research Institute of State Forestry Administration, Beijing 100029, \\ China \\ ${ }^{3}$ Beijing Forestry University, Beijing 100083, China \\ awoodtesting@163.com, bfuwansi@126.com, cxiaojiang56@126.com, dyumiaoljs@163.com
}

\begin{abstract}
Keywords: Lumber, surface defects, knot, image processing, morphology
Abstract. Knot is the commonest surface defect, which has the greatest impact on lumber. A image processing method was designed using Matlab image processing toolbox in this paper, which combined with mathematical morphology theory. Based on plenty of experiments, two turning coordinate points of piecewise linear transformation, $(0.35,0.2)$ and $(0.75,0.9)$, were selected, and a formula to calculate the threshold value of binarization were designed. The image processing method produces the desired effect, can accurately detect the obvious knots defects.
\end{abstract}

\section{Introduction}

Surface defects (such as knots, cracks, poles) will seriously affect the quality of the lumber, change its normal performance, and result in ineffectiveness [1,2]. Among many lumber surface defects, knot is the commonest one and has the greatest impact on the lumber quality. Based on the computer image processing technology and the mathematical morphology theory, this paper is trying to put forward an automatic detection algorithm of the knots on the lumber surface. Also, the Matlab image processing tools is applied in the knots image test experiments to verify the knots image preprocessing methods.

Mathematical morphology is a nonlinear branch of the signal processing field and concerns the application of set theory of image analysis [3]. Morphology refers to the study of shapes and structures from a general scientific perspective [4]. Swelling and corrosion are two basic operations in mathematical morphology, based on which a variety of complex operations, such as opening, closing, etc. can be composed [5]. Based on these basic operations some mathematical derivation and practical algorithm can also be combined, which can be analyzed and processed for the shape and structure of the image processing, including image segmentation, feature extraction, edge detection, image filtering, image enhancement and restoration, etc. .

Simple erosion is a process to eliminate all the boundary points of an object, with the result that the rest of the object along the periphery of a pixel becomes smaller than the original area of the object. If the object is circular, after every erosion, its diameter will reduce two pixels; in any direction at a point on the object, if the pixel is less than three dilation, then the object will split from the point into two objects after the first erosion. Simple dilation is a process to merge all the contact points connected with the background of an object into the object. The result of the process is to increase the area of the object and the corresponding number of points. If the object is a circle, its diameter increases two pixels after every time of dilation. If the point in any direction of the two objects is less than three pixels, it will link up at that point.

Erosion can eliminate small areas of noise in an image, dilation can fill the hollow. Give image first erosion and then dilation operation is called open operation. This can eliminate small objects, isolate objects at slender points, change the area when smooth borders of larger objects. If an image is processed first the expansion and then shrink, we call closing operation. This can fill tiny holes of objects, connect nearby objects, and smooth its boundaries without significantly changing the object area. Typically, when the image with noise goes binary process using threshold value, the resulting boundary is very rough, the object region has some misjudged holes, the background area of scatters 
small noise objects. Continuous opening and closing operation can obviously improve the situation. We can produce the desired effect after connect several erosion plus the same number of dilation.

\section{Experimental Procedure}

\section{Image processing flowchart}

The designed processing flowchart of lumber surface defect image is shown in Figure1. It includes: Image gray processing, gray-scale transformation, image smoothing, image segmentation, morphological processing, and edge detection. The purpose and effect of each processing step will be illustrated with the following examples.

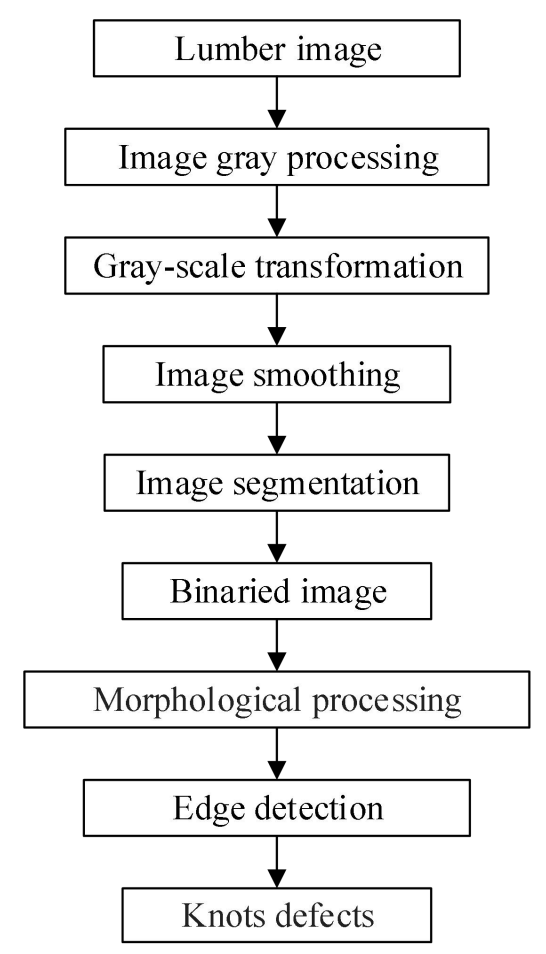

Figure 1. Flowchart of the surface image recognition algorithm

\section{Experimental samples}

Four representative knots defect images of Larch were selected and shown in Figure 2. This images were JPG format and taken by a digital camera, with resolution of $600 \times 800$.
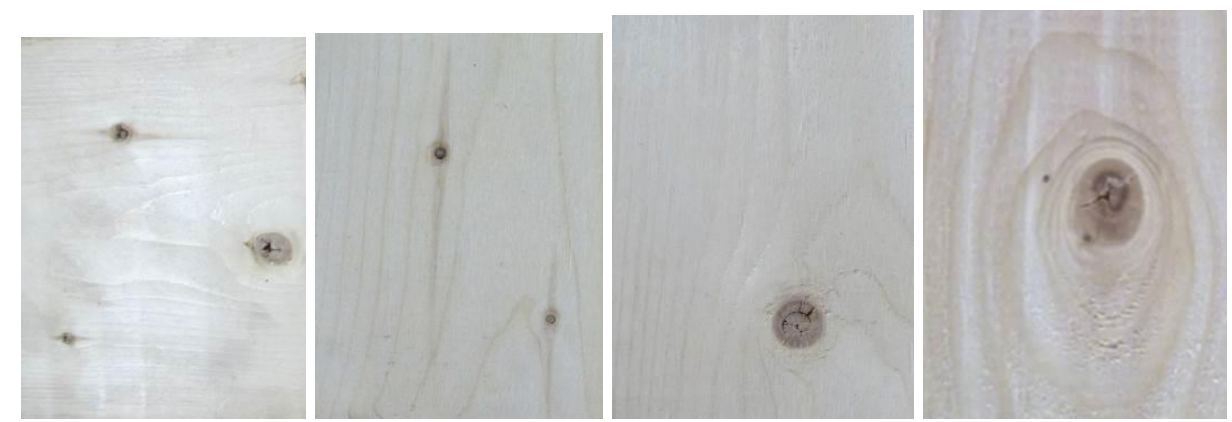

Figure 2. Knots defect images 


\section{Gray-scale image}

The process to transform the color image into a grayscale image was called gray image processing. In order to reduce the difficulty of image processing, the color RGB image was converted into a grayscale image. In this paper, a weighted average of the RGB image of the three components was used to calculate gray value as shown in formula (1). Wherein the Gray means gray value, R, G, B are red, green and blue component values of image. The gray-scale images are shown in Figure 3.

Gray $=0.3 * \mathrm{R}+0.59 * \mathrm{G}+0.11 * \mathrm{~B}$
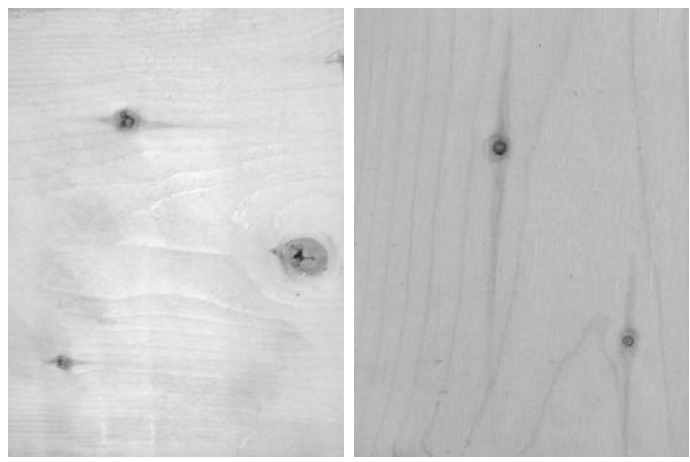
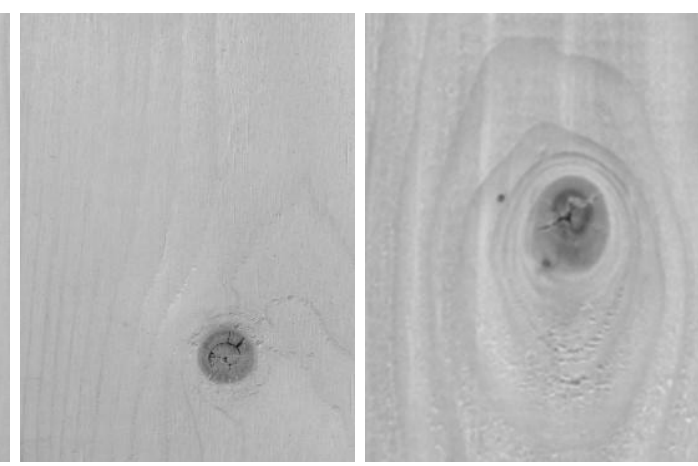

Figure 3. Gray-scale images

\section{Gray-scale transformed}

General imaging system only has a certain lightness response range, often has insufficient contrast, which ends up with poor visual effect. Gray-scale transformation is an important means of image enhancement. It can expand the dynamic range of the image, increase contrast, make images clearer, and with more obvious features. The piecewise linear method was used to highlight the target range without lose other details on the gray scale. Two turning points chosen herein coordinates $(0.35,0.2)$ and $(0.75,0.9)$, the transformed images are shown in Figure 4 . The figure shows the contrast enhanced significantly after conversion.

\section{Image smoothing}

The main purpose of image smoothing is to reduce the image noise [6]. Image noise comes from many resources, electromagnetic waves, high frequency noise interference from outside the system; but also from those within the system, such as thermal noise, mechanical jitter noise interference. Due to interference, the actual images obtained contain noise. The method to reduce noise was filtering. In this paper, $5 \times 5$ (median filter) and $7 \times 7$ (standard deviation) combined with Gaussian filter were used to de-noise gray image smoothly. The smoothing images are shown in Figure 5. Some of the glitches of unobvious texture were well inhibited.

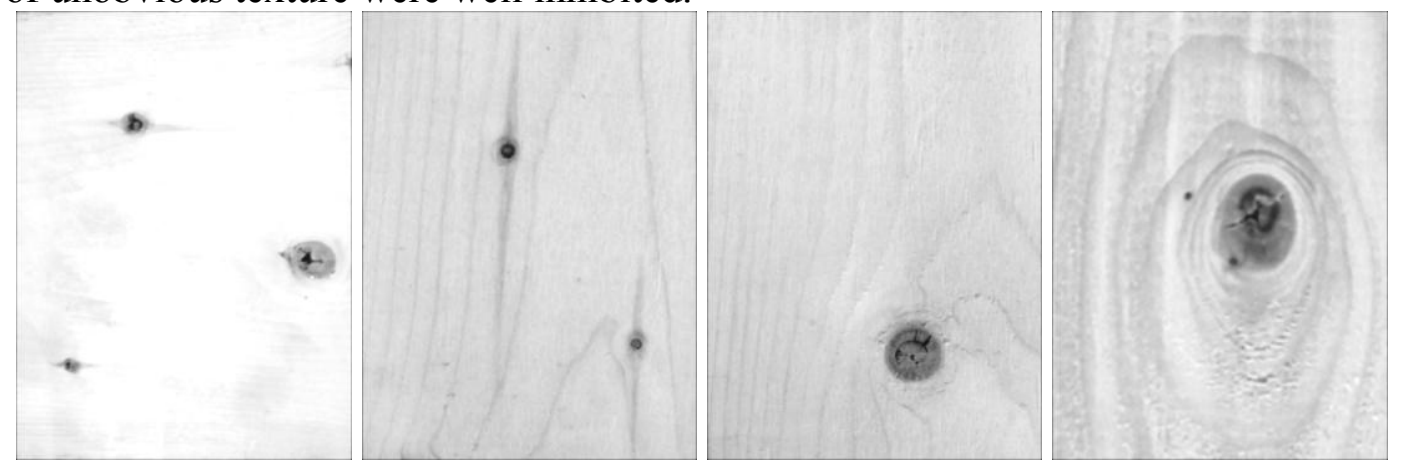

Figure 4. Gray-scale transformed images 


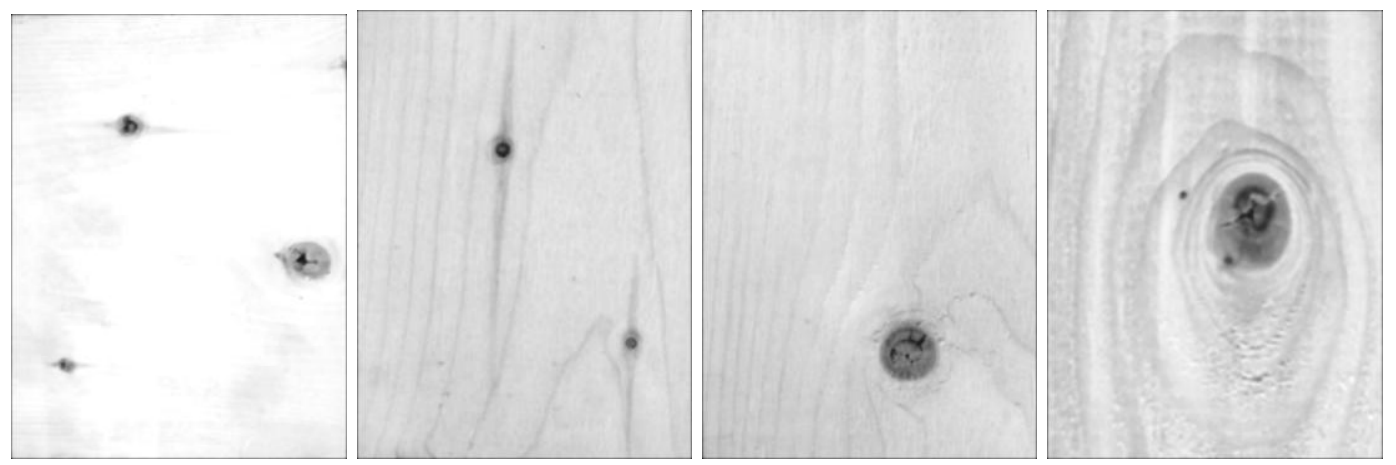

Figure 5. Smoothing images

\section{Image segmentation}

Image segmentation is one of the most important steps for the analysis of processed image data. Its main goal is to divide an image into parts that have a strong correlation with objects or areas of the real world contained in the image [7]. Gray-level thresholding is the simplest segmentation method. Many objects or image region are constant reflectivity or light absorption of their surfaces; so that a brightness constant or threshold can be determined to segment the objects from the background. Thresholding is computationally inexpensive and fast. In fact, it is the oldest segmentation method and is still widely used in simple application $[8,9]$. Through a lot of experiments, the formula (2) were used to calculate the threshold value of binarization. Segmented binary images are shown in Figure 6.

$$
\text { Thd }=\frac{M-V}{M+V} T h r
$$

Where Thd is the final threshold, Thr is the primary value calculated through the largest class variance method, $\mathrm{V}$ is the variance of grayscale images, $\mathrm{M}$ is the average value of gray-scale image.

\section{Morphological processing}

In this paper, the binary morphological image processing carried out include opening operation, closing operation, remove isolated foreground pixels, filling single pixel holes, etc. Morphological processed images are shown in Figure 7. Some of the smaller isolated interference points shown were eliminated after treatment, the edges of the image becomes rounder.

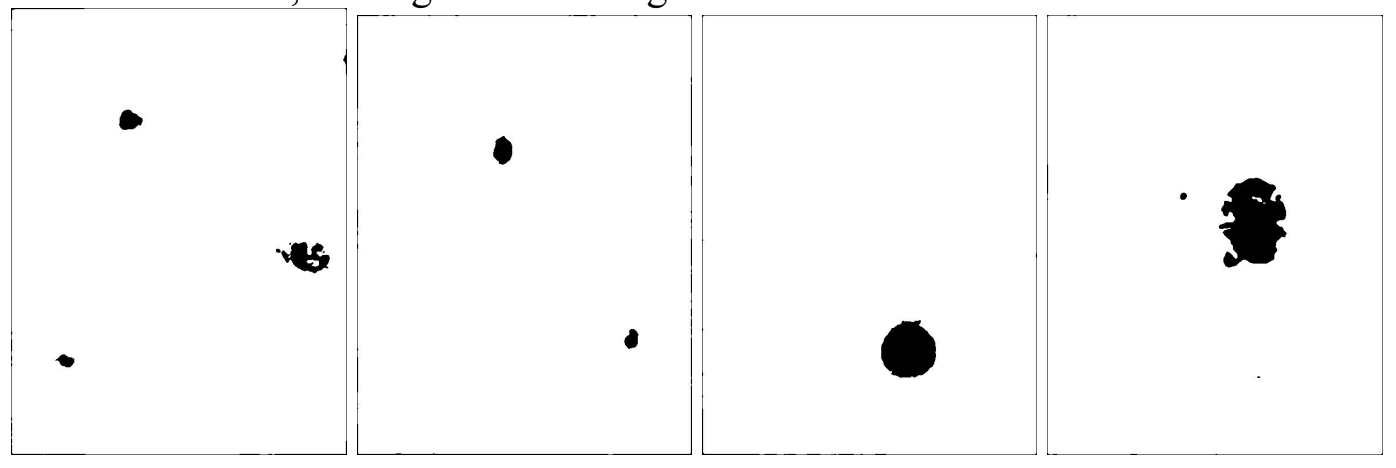

Figure 6. Segmented images 


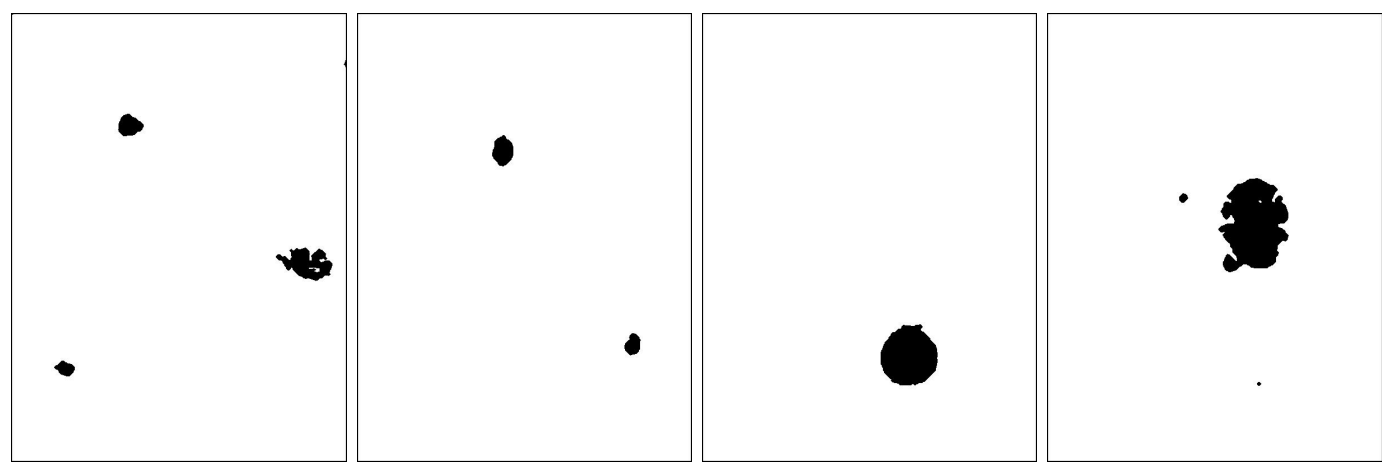

Figure 7. Morphological processed images

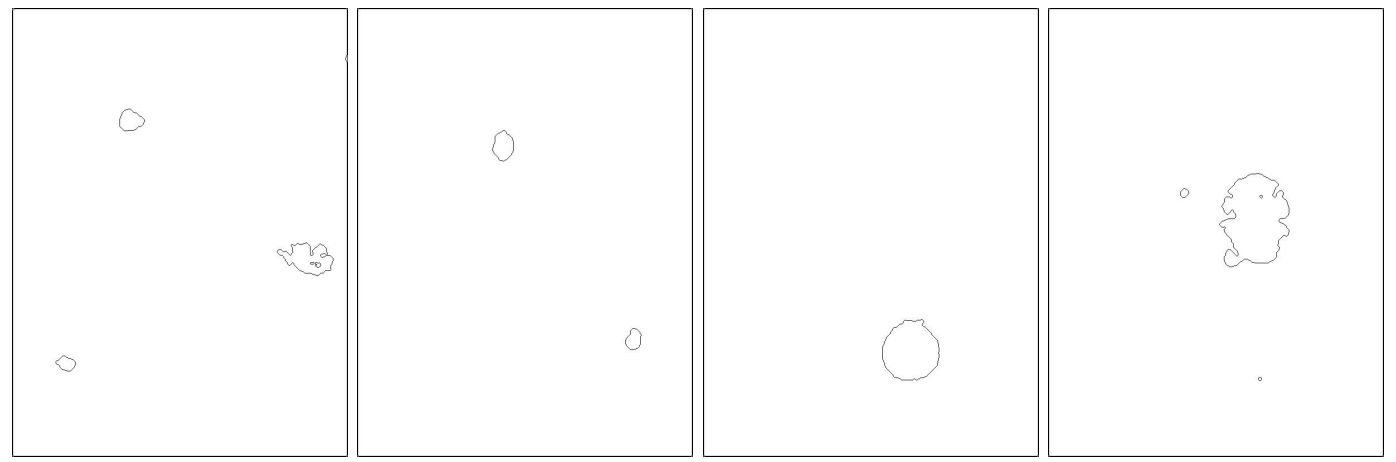

Figure 8. Defects outline images
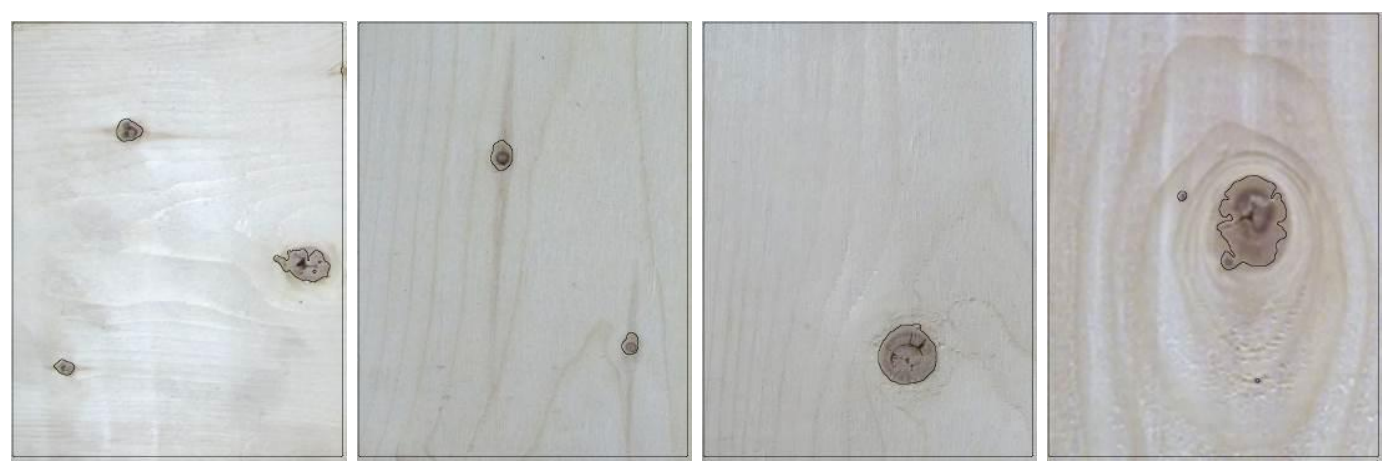

Figure 9. Suspected defect extraction of images

\section{Edge Detection}

Image defects outline is shown in Figure 8; contours of visible defects were smooth and clear. Figure 9 shows the superimposed effect image extracted from adding original contour defect (Fig.2). The defect had been completely extracted out; of course, some texture (not defects such as knots), a relatively deep color area, etc. had also been extracted, which required follow-up to identify whether they were the knots defects.

\section{Conclusions}

By selecting four typical knots defect image, this paper is based on image processing on gray-scale image, image smoothing, gray-scale transformation, image binarization, filtering, morphological processing, edge extraction and other computing experiments. Through observation, the image processing for defects of knots produces the desired effect, can accurately detect the obvious knots defects. Using image processing technology to achieve automatic detection of defects in lumber knots, accurately positioning of knots defects has important significance for statistical analysis, control, cut work. 


\section{Acknowledgments}

This work is supported by the Fundamental Research Funds for Central Public Welfare Research Institutes project, China (No. CAFINT2015C14).

\section{References}

[1] J. H. Yang, W. Zhang, W. Q. Hu, etc., China Patent 201110212438.2(2013)

[2] J. X. Yin, X. W. Lou, M. L. Huang: submitted to Journal of Zhejiang Forestry College (2008), in Chinese

[3] N. P. Brouwer, in: Image pre-processing to improve data matrix barcode read rates, University of New Hampshire, Hanover, New Hampshire, USA( 2013)

[4] K. Parvati, B. S. Prakasa Rao, and M. Mariya Das: submitted to Discrete Dynamics in Nature and Society(2008)

[5] Q. Q. Ruan: submitted to Publishing House of Electronics Industry (2005), in Chinese

[6] P. Pouladzadeh, in: An image processing and pattern analysis approach for food recognition, University of Ottawa, Parisa Pouladzadeh, Ottawa, Canada (2013)

[7] M. Sonka, V. Hlavac, R. Boyle, in: Image Processing, Analysis, and Machine Vision, 3th edn, Nelson Engineering Publisher (2008)

[8] D. Bajracharya: 'Real time pattern recognition in digital video with applications to safety in construction sites', University of Nevada, Las Vegas, USA (2013)

[9] J. Cheng, in: Automated detection and time lapse analysis of dendritic spines in laser scanning microscopy images, Northeastern University, Boston, Massachusetts, USA (2009) 